\title{
A Mobile Based Tigrigna Language Learning Tool
}

\author{
http://dx.doi.org/10.3991/ijim.v9i2.4322 \\ Hailay Kidu \\ University of Gondar, Gondar, Ethiopia
}

\begin{abstract}
Mobile learning (ML) refers to the use of mobile and handheld IT devices such as Personal Digital Assistants (PDAs), mobile telephones, laptops and tablet PC technologies, in teaching and learning. Mobile learning is a new form of learning, using mobile network and tools, expanding digital learning channel, gaining educational information, educational resources and educational services anytime, anywhere .Mobile phone is superior to a computer in portability. This technology also facilitates the learning by yourself process. Learning without teacher is easy in this scenario. The technology encourages learn anytime and anywhere. Learning a language is different from any other subject as it combines explicit learning of vocabulary and language rules with unconscious skills development in the fluent application of both these things. This can only happen when language learners are exposed in authentic language use for many hours every week and this is something that mobile technologies can easily support. This article focuses on "Mobile Based Tigrigna Language Learning Tool" and implemented a tool for learn it.
\end{abstract}

Index Terms-Mobile, Learning, Tigrigna and Language, Language learning

\section{INTRODUCTION}

In Ethiopia, Tigrinya is the third most spoken language and the "Tigray" are the third largest ethnic group, after the Oromo and Amhara. In Eritrea, Tigrigna is by far the most spoken language, and they represent $55 \%$ of the population. Tigrinian, Tigrinyan, ${ }^{[15]}$ is a Semitic language spoken in the Tigray Region of Ethiopia (its speakers there are called "Tigraway") by the Tigrinya people, where it has official status, and in central Eritrea, where it is one of the two main languages of Eritrea, and, and among groups of emigrants from these regions, including some of the Beta Israel now living in Israel. There is no generally agreed upon name for the people who speak Tigrinya. A native of Tigray is referred to in Tigrinya as tigrāwāy (male), tigrāweytī (female), tigrāwōt or tegaru (plural)[1]. In Eritrea, Tigrinya speakers are officially known as the Bihér-Tigrigna which means nation of Tigrinya speakers. Bihér roughly means nation in the ethnic sense of the word in Tigrinya Muslim native Tigrigna speakers are known as the Jeberti, an Arabic name which implies conversion to Islam among Horn Africans. Tigrinya is the third most spoken language in Ethiopia, after Amharic and Oromo, and by far the most spoken in Eritrea. It is also spoken by large immigrant communities around the world, in countries including Sudan, Saudi Arabia, Germany, Italy, Sweden, the United Kingdom, Canada and the United States.
Currently many people visit Tigray - or hope to do so one day - because of the remarkable manner in which ancient historical traditions have been preserved. Therefore anybody who wishes to visit or stay in Tigray and in Eritrea can learn the language for communication in formal class from schools, colleges or Universities. But there is no why that provides learning out of the formal class [2][3].

Tigrigna is the third most spoken language in Ethiopia, after Amharic and Oromo, and by far the most spoken in Eritrea. It is also spoken by large immigrant communities around the world, in countries including Sudan, Saudi Arabia, Germany, Italy, Sweden, the United Kingdom, Canada and the United States. Any interested Non- Tigrigna speaking citizens may also learn. Tigrigna language consists of around 300 alphabets and the language itself is highly inflectional, from one root word we can drive many words with different meaning. Therefore learning this language will take considerable amount of time. Even though the use of mobile phones is growing, there is no work done on Tigrigna mobile learning Tool.

\section{RELATED WORK}

Tigray forms the northernmost reaches of Ethiopia, and is located between 36 degrees and 40 degrees east longitude. Its north-south extent spans 12 and half degrees to 15 degrees north. It is bordered by Eritrea in the north, Sudan to the west, Amhara to the southwest and afar in the east. It is a very historic region noted for its custodianship of one of the powerful civilizations of the ancient world, which was centered here from at least $400 \mathrm{BC}$ to the 10 th century AD. Spreading far beyond modern Tigray, it molded the earliest culture of Ethiopia and left many historical treasures towering finely carved stele, the remains of extensive palaces, and the ancient places of worship are still vibrant with culture and pageantry [15].

The name of the language is Tigrinya. Tigrinya is descended from an ancient Semiticlanguage called Ge'ez. The Ethiopian and Eritrean Orthodox Churches officially use the Ge'ez as a liturgical language today, as in the past. The Tigrinya language is the direct descendant of Ge'ez, unlike Amharic (thought to be descended from a specific dialect or cluster of dialects of $\mathrm{Ge}^{\prime} \mathrm{ez}$ ) and other southern Ethiopian Semitic languages[7][8][9]. In Ethiopia, Tigrinya is the third most spoken language and the "Tigray" are the third largest ethnic group, after the Oromo and Amhara. In Eritrea, Tigrigna is by far the most spoken language, and they represent $55 \%$ of the population. Tigrinian, Tigrinyan, is a Semitic language spoken in the Tigray Region of Ethiopia (its speakers there are called "Tigraway") by the Tigrinya people, where it has official status, and in 
central Eritrea, where it is one of the two main languages of Eritrea, and, and among groups of emigrants from these regions, including some of the Beta Israel now living in Israel. There is no generally agreed upon name for the people who speak Tigrinya.

A native of Tigray is referred to in Tigrinya as tigrāwāy (male), tigrāweytī (female), tigrāwōt or tegaru (plural). In Eritrea, Tigrinya speakers are officially known as the Bihér-Tigrigna which means nation of Tigrinya speakers. Bihér roughly means nation in the ethnic sense of the word in Tigrinya Muslim native Tigrigna speakers are known as the Jeberti, an Arabic name which implies conversion to Islam among Horn Africans. Tigrinya is the third most spoken language in Ethiopia, after Amharic and Oromo, and by far the most spoken in Eritrea. It is also spoken by large immigrant communities around the world, in countries including Sudan, Saudi Arabia, Germany, Italy, Sweden, the United Kingdom, Canada and the United States. Currently many people visit Tigray - or hope to do so one day - because of the remarkable manner in which ancient historical traditions have been preserved[10][11]. Therefore anybody who wishes to visit or stay in Tigray and in Eritrea can learn the language for communication in formal class from schools, colleges or Universities. But there is no why that provides learning out of the formal class.

Tigrigna is the third most spoken language in Ethiopia, after Amharic and Oromo, and by far the most spoken in Eritrea. It is also spoken by large immigrant communities around the world, in countries including Sudan, Saudi Arabia, Germany, Italy, Sweden, the United Kingdom, Canada and the United States[14][16][17]. Any interested Non- Tigrigna speaking citizens may also learn. Tigrigna language consists of around 300 alphabets and the language itself is highly inflectional, from one root word we can drive many words with different meaning. Therefore learning this language will take considerable amount of time. Even though the use of mobile phones is growing, there is no work done on Tigrigna mobile learning Tool [12][13].

\section{PROPOSED WORK}

The proposed system has a client and the server application. A user with android mobile phone will be using this Tigrigna language Learning tool to practice basic skills of the language. He/she can learn the Alphabets of the language which helps for reading and writing, can also learn vocabulary words and different recorded audios will be used to help the user develop his/her listening skills. The tool can be used by a foreigner or anybody whose first language is not Tigrigna. To develop this application we use android 2.2(API) with SDK platforms as it provides the tools APIs necessarily to begin developing android applications on the android platform using android java programming language. It includes mobile device emulator- a virtual android operating system mobile device that runs on your computer. The emulator lets you develop and test android applications without using a physical android device. For editing and developing the user interface we use eclipse IDE since the tool is designed to give you a powerful and integrated environment in which to build android applications.

For the database we use the Built in SQL it that lets you store the user profiles in the database. The system shall be available for the learner as long as the learner mobile is working properly. The security of the system is related to the security of the mobile phone. Mobile phone security mechanisms such as pin code and password protection can also be used as security protection. The system is developed using object oriented approach and, therefore, adding new functionality or modifying some parts of the system will not affect the other parts of the system. The system is developed with easy to use and interactive user interface. Since the system runs on a mobile phone for better management of the small screen, the right type and amount of interactive user interfaces are used. In this future based on the learners selection the application will display all the lessons of Tigrigna alphabets. The Learner can view all the details of the alphabets in two row format user can select the second row of the alphabet then it will be displayed the sequence of the alphabets to the first row. When the learner clicks each alphabet button the learner can listen for the corresponding sound. In this future based on the learners selection the application will display all the lessons of Tigrigna words under the words tutorials are different sub category of tutorials. The Learner can view all the details of the words in three column table format the first column indicates the picture of the word the second column shows Tigrigna meaning of the word the third column shows equivalent meaning of English. When the learner clicks Tigrigna meaning of the word he/she listen text to audio synthesizer pronunciation. In this future based on the learners selection the application displays different options from the common Tigrigna conversations: like greetings, introduce one self, and leave taking under the sub categories there are different similar tutorials. The same to alphabet tutorials the corresponding sound will be displayed.

\section{A. System Implementation}

The Android application is developed by using Eclipse Helios Integrated Development Environment (IDE). Android SDK which includes a variety of custom tools that help us develop mobile applications on the Android platform is used. Android Emulator and ADT plug-in for Eclipse are the most important of these tools. ADT extends the capabilities of Eclipse to quickly set up new Android projects, create an application UI, add components based on the Android Framework API, debug applications using the Android SDK tools, and even export signed (or unsigned) .apk files in order to distribute application.

The GUI is designed using XML. Separating the user interface part from java code helps to easily modify the application without touching the java code. The sound files are stored on the sdcard of the emulator by pushing in to the emulator because the if the size of the application large it may be difficult to install on the device due to memory capacity. A total of 90 working hours spanning over 3 months have been spent on designing and developing this application and an approximate of additional 5 hours have been spent on testing the application for its correct functionality after the application has been fully developed.

Debugging of the application throughout the development is done using Dalvik Debug Monitor Server (DDMS). DDMS provides port-forwarding services, screen capture on the device, thread and heap information on the device, logcat, process, and radio state information, 
incoming call and SMS spoofing, location data spoofing etc. ${ }^{[22]}$ Implementation of each feature in this application is explained in detail in the system description part. The basic reasons to choose Android platform, for this project, are the following:

- It uses free type, a free and open source bitmap and vector font engine, which supports Unicode text layout and glyph rendering. Ethiopic Unicode fonts, such as Microsoft's Nyala and GFZ's Geez Free Zemen, can be used to render text using free type on the Android platform. Other platforms such as the Palm OS lack the internationalization support required to fully meet the needs of Ethiopic software development.

- It is based on open source product, Linux kernel, which brings a big change from the current oligopoly of mobile OS makers that charge handset manufacturers various licensing fees.

- It comes with immediate availability to mobile versions of Google search, Google Maps, and Google Products that allow independent developers to create their own array of applications at a much less restrictive cost.

Eclipse version Helios is used as integrated development environment. In keeping with the Open Handset Alliance's theme of truly opening the mobile development market, Eclipse is one of the most fully featured, free, Java IDEs available. Eclipse is also very easy to use. The Open Handset Alliance has released an Android plug-in for Eclipse that allows Creating Android-specific applications, compiling them, and using the Android Emulator to run and debug them.

In this section several screen shots of android emulator have been inserted to show the working of different features in the application.

The Home screen of the system, i.e. the Tigrigna Language Learning Tool topics are illustrated in figure 2. This is the first screen that appears after the learner selects the Tigrigna icon from his/her mobile device. It contains the main Topics of the tutorials and exercises. The user can also view his/her profile by selecting view profile from the home screen of the application menu. Each of the menu option has a list of their own sub menus which includes different lessons.

\section{CONCLUSION AND FuturE ENHANCEMENT}

The Goal of this project is to build an android application which helps in learning Tigrigna language that allowed learners to learn the language anywhere at any time informally. The tool is developed using Eclipse IDE for Java, Version: Indigo Service Release 1, in mind about the design standards and maintainability of the code. Foreigners who want to visit Tigray or Eretria can use this application for communication. Mobile application promotes students to use foreign languages most frequently and also keep in touch with the culture. The intention of teaching outside the class room can be accomplished by making use of this application. Since the application can use in work areas or in home. The application has wide scope for other mobile operating system developers and client and server based application developers. As a future work, this learning tool has a vast enhancement opportunity: First, the language learning tool can be extended to other lan-

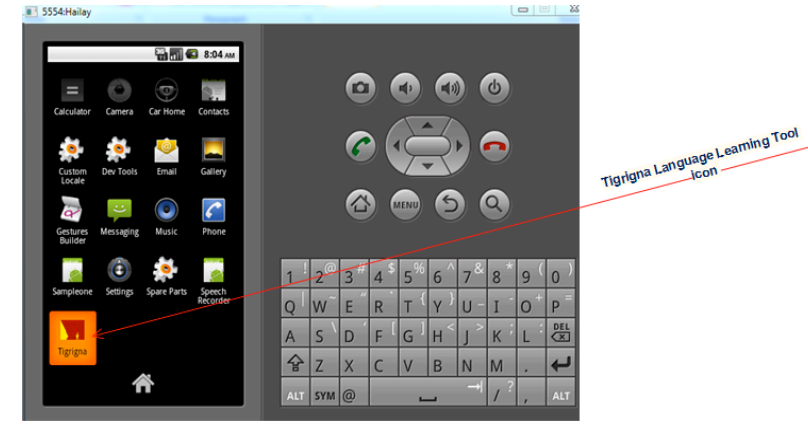

Figure 1. is the Android mobile home screen from which the Tigrigna Language Learning Tool is launched with the appropriate icon. I choose this icon because that icon is the people's flag so any one can easily identify it.

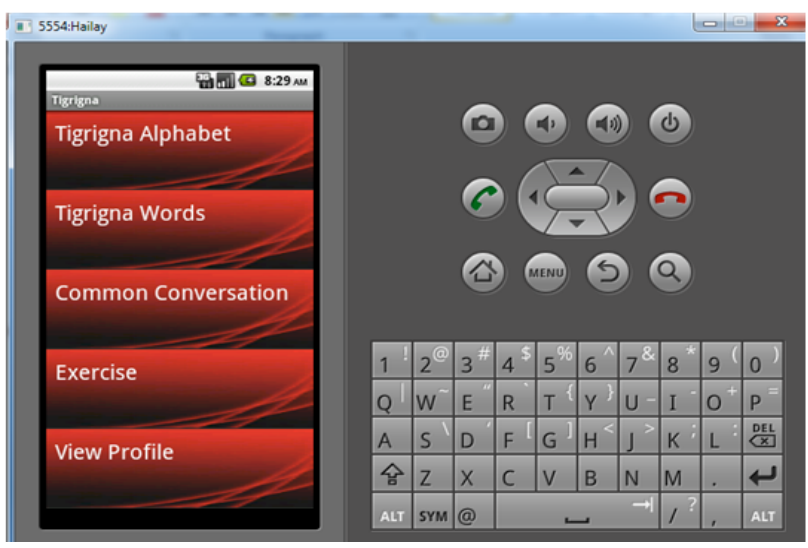

Figure 2. Home Screen of Tigrigna Language Learning Tool Application.

guages that are found in Tigray, Ethiopia, Eretria. Second, we can also design it in a client-server manner so that new lessons can be added to the system and the learner can download it whenever it is possible and use it next time. Third, since the tool uses a text to speech method to teach the words we can incorporate the best text-to -speech methods for Tigrigna language in the future. Fourth, this tool works only on android mobile phone therefore we can also extend it to other mobile phone platforms

\section{REFERENCES}

[1] Anastasios A. (2008) Requirements of Mobile Learning Applications. International Journal of Innovation and Learning, International Journal of Innovation and Learning. 5 (5): pp 457 - 479. http://dx.doi.org/10.1504/IJIL.2008.018043

[2] Jill A. and Carol S. (2004) learning with mobile devices. Learning and Skills Development Agency.

[3] Xinghong L. ( 2009) Applied Research on the Mobile Learning in Foreign Language, Second International Conference on Education Technology and Training, pp 309-312.

[4] Morita M.( 2003) The Mobile-based Learning (MBL) in Japan, proceedings of the First Conference on Creating, Connecting and Collaborating through computing. pp 203-270. http://dx.doi.org/10.1109/C5.2003.1222348

[5] Kong S., Ogata .H, Arnseth. H., Chan C., Hirashima T., and Klett F. (2009). Proceedings of the 17th International Conference on Computers in Education [CDROM]. Hong Kong: Asia-Pacific Society for Computers in Education. pp 325-525.

[6] Axumite T. (2011) Mobile Based Amharic Language Learning Tool. Addis Ababa University, Addis Ababa, Ethiopia, pp 1.

[7] Klaus R., Judith S., Norbert P., Graham A. ( 2011) Mobile learning: Crossing boundaries in convergent environments. ISSN 17533385 . 


\section{PAPER}

A Mobile Based Tigrigna Language Learning ToOL

[8] Vivian O. (2010) SMS-Based Mobile Learning System: A Veritable Tool for English Language Education in Rural Nigeria. Postgraduate strand at ICTD. National Open University of Nigeria. pp $1-2$

[9] Cui Y. and Bull S. (2005)Context and learner modeling for the mobile foreign language learner pp 353-367.

[10] Sara R., Shelley S.(2010) Design and Implementation of a mobile game based system to Support Chinese language speaking for International Students in a Chinese Environment.National Tsing Hua University. pp 428-430.

[11] Xinghong L. ( 2009) Applied Research on the Mobile Learning in Foreign Language. Second International Conference on Education Technology and Training. Pp 309-312.

[12] Milton J. (2006) Literature Review in Languages, Technology and Learning, Centre for Applied Language Studies. University of Wales Swansea pp 6.

[13] Maria L. and Vinci D. (2004) Possibilities of application of e-tools in education: mobile learning, Florence, Italy. Pp 200-2022.

[14] http://www.fotosearch.com/image-source/raw-foods-1/IGS653/ (last accessed on March 20,2012).

[15] http://www.absoluteastronomy.com/topics/TigrayTigrinya_people (last accessed April 8,2012).

[16] James F. (2008) A Programmer's Guide ,The McGraw-Hill Companies, pp 11 .

\section{AUTHOR}

Hailay Kidu is a Lecturer, Researcher and software developer at the Department of Information Technology, University of Gondar. He received his first degree in 2007 by Information Technology, from Mekelle University, Masters Degree in 2012 by Information Technology from University of madras. He got special training in Leader- ship and Management Skills Development Training Program from Vision Ethiopian Congress for Democracy (VECOD), In ICT Business Incubation center from Mekelle Institute of Technology (MIT), In Solar Photovoltaic System (PV) from University of TUDelft. He was worked in the National software development Project called National Integrated Justice Information System (NIJIS) that was undertaken by cyber soft, Ethiopian software companies and chain's ZTE companies for the period of may 2012 up to December 2012. Mr. Hailay kidu is a member of different professional associations including the Information Communication Technology since 2012, and the Association of Ethiopian Teachers since 2001 and Tigray Academic and Scientific network since 2012.

Currently Mr. Hailay kidu is a senior Lecturer researcher and application developer at the University of Gondar, Gondar, ethiopia.He is teaching in the area of software engineering and theoretical computer science. Information Technology Department University of Gondar, p.o.box 196,Mobile, +251920253449, (e-mail: hailiyekidu@gmail.com )

This work was an individual work and supported by the University of Gondar of Higher Education (MOHE) for the provision of the Prototype Research Grant Scheme 2013. This is a thematic research, addresses a national priority problem with clear potential for economic benefit (commercialization or have significance for the social or cultural benefit of societies). Submitted 1 September 2013. Published as resubmitted by the author 23 March 2015. 